\title{
PERIODIC SOLUTIONS OF SYSTEMS WITH ASYMPTOTICALLY EVEN NONLINEARITIES ${ }^{1}$
}

\author{
PETER E. KLOEDEN \\ Johann Wolfgang Goethe-Universität \\ Fachbereich Mathematik \\ D-60054 Frankfurt am Main, Germany \\ e-mail: kloeden@math.uni-frankfurt.de \\ ALEXANDER M. KRASNOSEL'SKII \\ Institute of Information Transmission Problems \\ 19 Bolshoi Karetny Lane \\ Moscow 101447, Russia \\ e-mail:amk@ippi.ac.msk.su
}

(Received July, 1998; Revised January, 1999)

\begin{abstract}
New conditions of solvability based on a general theorem on the calculation of the index at infinity for vector fields that have degenerate principal linear part as well as degenerate "next order" terms are obtained for the $2 \pi$-periodic problem for the scalar equation $x^{\prime \prime}+n^{2} x=g(|x|)+f(t, x)+$ $b(t)$ with bounded $g(u)$ and $f(t, x) \rightarrow 0$ as $|x| \rightarrow 0$. The result is also applied to the solvability of a two-point boundary value problem and to resonant problems for equations arising in control theory.
\end{abstract}

Key words: Index at Infinity, Degenerate Linear Part, Asymptotically Even Nonlinearity, Boundary Value Problems.

AMS subject classifications: $47 \mathrm{H} 11,47 \mathrm{H} 30$.

\section{Introduction}

Consider the problem of the existence of a $2 \pi$-periodic solution of a forced second order quasilinear equation $x^{\prime \prime}=w(t, x)$, such as the nonlinear forced pendulum equation $x^{\prime \prime}+a x=f(x)+b(t)$, and suppose that its right-hand side is asymptotically linear, i.e., the following limit exists:

$$
\lim _{x \rightarrow \infty} \frac{w(t, x)}{x}=-k
$$

\footnotetext{
${ }^{1}$ P.E. Kloeden was partially supported by the Australian Research Council Grant A 89132609 and A.M. Krasnosel'skii by Grants 97-01-00692 and 96-15-96048 of the Russian Foundation of Fundamental Research.
} 
If $k$ is not the square of an integer, then the problem is not resonant and it can be easily investigated by, for example, the Schauder principle. Suppose instead that $k=n^{2}$ and $w(t, x)=-n^{2} x+v(t, x)$ with bounded $v(t, x)$. The usual way to study this case is to assume the Landesman-Lazer conditions, i.e., $v(t, x) \rightarrow V^{ \pm}(t)$ as $V^{ \pm}(t)$, the problem can be handled by, for example, topological methods. If these non-degeneracy conditions fail, then it is necessary to use properties of terms that vanish at infinity (see [5]).

In this paper we consider a situation without the Landesman-Lazer property: the $2 \pi$-periodic problem for the equation $x^{\prime \prime}+n^{2} x=g(|x|)+f(t, x)+b(t)$ where the bounded function $g(u)$ does not have a limit at infinity and $f(t, x)$ tends to zero as $|x| \rightarrow \infty$.

The $2 \pi$-periodic problem for linearized equation $x^{\prime \prime}+n^{2} x=0$ is degenerate for an integer $n$ and the behavior of perturbations of this equation depends on some delicate properties of the bounded nonlinearities $g(u)$ and $f(t, x)$. If $g(u) \equiv 0$ and $f(t, x) \equiv 0$ then the Fredholm alternative gives a complete answer on the solvability of the $w \pi$ periodic problem of $x^{\prime \prime}=n^{2} x=b(t)$ in terms of the value of the complex number

$$
b_{n}=\int_{0}^{2 \pi} e^{i n t} b(t) d t .
$$

If $b_{n}=0$, then there exists a 2-dimensional linear set of $2 \pi$-periodic solutions, while if $b_{n} \neq 0$, then such solutions do not exist.

Nonlinear terms change the situation. Suppose that we know only the asymptotics of the nonlinearities at infinity, i.e. the behavior of the nonlinearities for sufficiently large $|x|$. For example, consider the equation $x^{\prime \prime}+n^{2} x=f(t, x)+b(t)$ with $f(t, x) \rightarrow 0$ as $|x| \rightarrow \infty$. If $b_{n} \neq 0$, then the topological properties of the equations cannot guarantee the solvability of the $2 \pi$-periodic problem; the index at infinity of the corresponding vector fields is equal to 0 . If $b_{n}=0$, then, under some appropriate hypotheses, the index differs from 0 and topological methods are applicable. Related problems (for $g(u) \equiv 0$ ) were studied in $[1,2]$, in series of papers by J. Mawhin and his co-authors (see $[1,3,7,8]$ and the references therein). In addition, the equation $x^{\prime \prime}+n^{2} x=g(|x|)+v(t, x)$ with a non-degenerate Landesman-Lazer type term $v(t, x)$ was considered in [4].

In the next section we present a new theorem on the solvability of our equation. This theorem follows from a general theorem, Theorem 2, on the calculation of the index at infinity that is formulated in Section 3. Proofs are given in Sections 4 and 5. Theorem 2 can also be applied to various problems on solvability and bifurcation at infinity, etc., for some other boundary value problems. Examples of possible applications to two-point boundary value problems and problems of forced oscillations in control systems are presented in Section 6.

\section{Main Result for the Second Order Equation}

Consider the equation

$$
x^{\prime \prime}+n^{2} x=g(|x|)+f(t, x)+b(t)
$$

with non-zero integer $n$ and functions $f(t, x)$ and $b(t)$ that are $2 \pi$-periodic in $t$. These 
functions together with the function $g(u)$ are supposed to be bounded and continuous with respect to all their variables. (This assumption can be weakened: It is possible to prove the assertion of Theorem 1 for Carathéodorian functions $f(t, x)$ and the integrable functions $b(t)$.) The following hypotheses are supposed to be valid.

$(A)$ One of the following one-sided estimates holds:

$$
f(t, x) \cdot \operatorname{sign} x \geq \varphi(|x|), \quad|x| \geq u_{0}
$$

or

$$
f(t, x) \cdot \operatorname{sign} x \leq-\varphi(|x|), \quad|x| \geq u_{0}
$$

for some $u_{0}>0$, where $\varphi(u):\left\{u \geq u_{0}\right\} \rightarrow \mathbb{R}^{+}$is a positive continuous nonincreasing function.

(B) An asymptotical Lipschitz condition holds: there is an $\alpha \in(0,1)$ such that

$$
|g(u)-g(v)| \leq c r^{-\alpha}|u-v|, \quad r=\min \{u, v\}, \quad u, v \geq u_{0} .
$$

Hypothesis $(B)$ is valid, for example, for $g(u)=\sin \left(u^{1-\alpha}\right)$. If the function $g(u)$ is differentiable, then this hypothesis is equivalent to

$$
\left|g^{\prime}(u)\right| \leq c u^{-\alpha}, \quad u \geq u_{0}
$$

Theorem 1: Let $b_{n}=0$, suppose that both hypotheses $(A)$ and $(B)$ hold, and let

$$
\lim _{u \rightarrow \infty} \varphi(u) u^{\alpha}=\infty
$$

Then (1) has at least one $2 \pi$-periodic solution.

From the proof of Theorem 1 in Section 5 one can see that the function $\varphi(|x|)$ in conditions (2) and (3) can be replaced by the function $\varphi_{0}(t,|x|)$, where

and mes $\Omega_{0}>0$.

$$
\varphi_{0}(t, u)=\left\{\begin{array}{cc}
\varphi(u), & t \in \Omega_{0}, \\
0, & t \notin \Omega_{0}
\end{array}\right.
$$

\section{An Abstract Generalization}

In this section we give an abstract generalization of Theorem 1.

Let $\Omega$ be some set of positive finite measure and let $L^{2}$ denote the Hilbert space of scalar square integrable functions $x(t): \Omega \rightarrow \mathbb{R}$ with the usual scalar product $(\cdot, \cdot)$ and norm $\|\cdot\|$.

We want to calculate the index at infinity (see, e.g. [6]) of the Hammerstein-type vector field

$$
\Upsilon x(\cdot)=x(\cdot)-A(x(\cdot)+f(\cdot, x(\cdot))+g(|x(\cdot)|)+b(\cdot)),
$$

in the space $L^{2}$, where $A$ is a completely continuous linear operator in $L^{2}$ and $f, g$ and $b$ are as above. If 1 is not an eigenvalue of the operator $A$, this index is defined 
and is equal $(-1)^{\sigma}$, where $\sigma$ is the sum of the multiplicities of all real eigenvalues of $A$ which are greater than 1 .

We will consider the case for which 1 is an eigenvalue of a normal linear operator $A$, that is with $A A^{*}=A^{*} A$. The normality of $A$ guarantees that there are no generalized eigenvectors corresponding to the eigenvalue 1 . Let $E_{0}=\operatorname{Ker}(I-A)$ denote the corresponding linear finite dimensional subspace of the eigenvectors of the eigenvalue 1.

The main restriction of the operator $A$ that allows vector fields with an even term $g(|x|)$ to be considered is the identity:

$$
\int_{\Omega} e(t), g(|e(t)|) d t=0, \quad e(t) \in E_{0}
$$

which was first mentioned in [4] and is valid for various important applications.

If $\Omega=[0,2 \pi]$ and $E_{0}$ is the 2-dimensional subspace containing the functions $\sin n t$ and $\cos n t$, then (6) is valid; this example occurs in the investigation of the $2 \pi$ periodic problem to be considered in Section 6 . The identity (6) is also valid for $\Omega=[0, \pi]$ and a 1-dimensional subspace $E_{0}$ containing the function $\sin n t$ for even $n$; this case arises in the study of degenerate two-point boundary value problems.

Suppose that

$\left(A^{\prime}\right)$ One of the following one-sided estimates

$$
f(t, x) \cdot \operatorname{sign} x \geq \varphi(t,|x|),|x| \geq u_{0}, \quad t \in \Omega
$$

or

$$
f(t, x) \cdot \operatorname{sign} x \leq-\varphi(t,|x|),|x| \geq u_{0}, \quad t \in \Omega
$$

for some $u_{0}>0$, holds where $\varphi(t, u): \Omega \times\left\{u \geq u_{0}\right\} \rightarrow \mathbb{R}^{+}$is a nonnegative Carathéodorian nonincreasing function which is strictly positive for $t \in \Omega_{0}$ with mes $\Omega_{0}>0$.

$\left(B^{\prime}\right)$ The asymptotical Lipschitz condition

$$
|g(u)-g(v)| \leq d(r)|u-v|, \quad r=\min \{|u|,|v|\}, u, v \geq u_{0}
$$

holds, where $d(r): \mathbb{R}^{+} \rightarrow \mathbb{R}^{+}$is some positive nonincreasing function satisfying

$$
\lim _{r \rightarrow \infty} d(r)=0
$$

The distribution function

$$
\chi(\delta)=\chi(\delta ; e)=\operatorname{mes}\{t \in \Omega:|e(t)| \leq \delta\}
$$

of a non-zero function $e(t) \in E_{0}$ plays an important role in the formulation and proof of the following theorem. Let $P$ denote the orthogonal projector onto $E_{0}$ and in formula (13) below, let $d(u)=d\left(u_{0}\right)$ for $0 \leq u<u_{0}$. that

Theorem 2: Let $P b(t)=0$. Suppose that both hypotheses $\left(A^{\prime}\right)$ and $\left(B^{\prime}\right)$ hold and

$$
\chi(0)=\operatorname{mes}\{t \in \Omega: e(t)=0\}=0 .
$$


for any non-zero function $e(t) \in E_{0}$. Suppose also that the operator $A$ maps square integrable functions into essentially bounded ones and that $A$ is continuous as an operator from the space $L^{2}$ into the space $L^{\infty}$. Moreover, suppose for any $R>0$ and $u_{*} \geq u_{0}$ that

$$
\lim _{\xi \rightarrow \infty} \sup _{e \in E_{0},\|e\|=1} \frac{\int_{\Omega}|e(t)| d(\xi \mid e(t \mid) d t}{\int_{\Omega}|e(t)| \varphi\left(t, u_{*}+R \xi|e(t)|\right) d t}=0
$$

and

$$
\lim _{\xi \rightarrow \infty} \sup _{e \in E_{0},\|e\|=1} \frac{\chi\left(\xi^{-1}, e\right)}{\int_{\Omega}|e(t)| \varphi\left(t, u_{*}+R \xi|e(t)|\right) d t}=0 .
$$

Then

$$
\operatorname{ind}_{\infty} \Upsilon=(-1)^{\sigma_{0}}
$$

with $\sigma_{0}=\sigma+\operatorname{dim} E_{0}$ for the case (7), and $\sigma_{0}=\sigma$ for the case (8), where $\sigma$ is the sum of the multiplicities of all real eigenvalues of the operator $A$ that are greater than 1 .

Condition (12) has been used by many authors, while the functions (11) were considered in a related context in Chapter 25 of [1] and were later used systematically in [3]. The combination of (6) with the condition that $f(t, x) \rightarrow 0$ has not been considered before. The assumption about the operator $A: L^{2} \rightarrow L^{\infty}$ is technical and can be omitted, but this then makes proof much more cumbersome. It is usually valid in many applications.

In specific examples, conditions (13) and (14) are often not as awkward (see [3]) as may at first seem, e.g. in the case of Theorem 1, the relation (5) guarantees both (13) and (14). If the function $d(u)$ has the form $d(u)=c u^{-\alpha}$ for some $\alpha \in(0,1)$ and (14) holds, then (13) is equivalent to

$$
\lim _{\xi \rightarrow \infty} \sup _{e \in E_{0},\|e\|=1} \frac{\xi^{-\alpha}}{\int_{\Omega}|e(t)| \varphi\left(t, u_{*}+R \xi|e(t)|\right) d t}=0 .
$$

\section{Proof of Theorem 2}

Consider the homotopy

$$
\Phi(x, \lambda)=\Upsilon x+\lambda A x
$$

where $\lambda$ has a fixed sign. Let us prove for $|\lambda| \leq \lambda_{0}$ with positive $\lambda_{0}$ small enough that the vector field $\Phi(x, \lambda)$ is non-zero for sufficiently large values of $\|x\|$ under the assumptions of Theorem 2. We consider only non-positive values of $\lambda$ for the case where (7) holds and non-negative $\lambda$ for the opposite case.

This is a prior estimate will prove Theorem 2: for small $\lambda \neq 0$, the linear part $I-(1-\lambda) A$ of the field $\Phi(x, \lambda)$ is non-degenerate and its index at infinity has exactly the value given in Theorem 2 ; the number $\mu=(1-\lambda)^{-1}$ is an eigenvalue of the operator $(1-\lambda) A$ with $\mu>1$ iff $\lambda<0$. The general properties of the index then complete the proof. The proof of a common estimate will be given only for the case (7), $\lambda \in$ $\left[-\lambda_{0}, 0\right]$ 
Suppose that $\Phi(x, \lambda)=0$ for some $x \in L_{2}$ and $\lambda \in\left[0, \lambda_{0}\right]$. Denote by $E_{1} \subset L^{2}$ the orthogonal to $E_{0}$ subspace and let $Q=I-P$. The projectors $P$ and $Q$ commute with the operator $A$ and the projector $P$ can be easily represented as $P x=$ $\sum\left(e_{j}, x\right) e_{j}$, where $\left\{e_{j}\right\}$ is an orthonormal basis in $E_{0}$ and $A P=P$.

For $|\lambda|$ small enough, the linear operators $B(\lambda)=Q(I-(1-\lambda) A)$ are continuously invertible in $E_{1}$ for any $\lambda$. Moreover, these inverse operators have uniformly bounded norms

$$
\left\|B(\lambda)^{-1}\right\|_{E_{1} \rightarrow E_{1}} \leq c_{1}, \quad|\lambda| \leq \lambda_{0}
$$

Here and below $c_{j}$ denote constants for which only the existence and not their exact value is of importance. In particular, these constants do not depend on $\lambda$ and $x$.

The equation $\Phi(x, \lambda)=0$ can be rewritten as the pair of equations: $Q^{\Phi}(x, \lambda)=0$ and $P \Phi(x, \lambda)=0$.

The first equation, rewritten as $B(\lambda) x=A Q(f(t, x)+g(|x|)+b(t))$, together with the continuity of the operator $A: L^{2} \rightarrow L^{\infty}$ implies that

$$
\|Q x\|_{L^{\infty}} \leq c_{2}
$$

The second equation can be rewritten as

$$
\lambda P x=P(f(t, x)+g(|x|))
$$

(recall that $P b=0$ is an assumption of Theorem 2). This last formula will now be considered in some detail

Let $P x=\xi e(t)$ where $\|e\|=1, \xi \geq 0$ and $h=Q x$. In view of (17) we have to obtain an a priori estimate for the scalar positive $\xi$.

Let us multiply (18) by $e(t)$. Then the scalar equality obtained has the form

which implies that

$$
\lambda \xi=\int_{\Omega} e(t)(f(t, x)+g(|x|)) d t
$$

$$
\int_{\Omega} e(t)(f(t, \xi e(t)+h(t))+g(|\xi e(t)+h(t)|)) d t \leq 0
$$

The scheme of the proof below is as follows. The left-hand side of (19) contains two terms. The first term can be estimated from below, the estimate having the form

$$
\begin{gathered}
\left.\int_{\Omega} e(t) f(t), \xi e(t)+h(t)\right) d t \\
\geq \int_{\Omega}|e(t)| \varphi\left(t, u_{*}+\xi|e(t)|\right) d t-c_{3} \chi\left(c_{3} \xi^{-1}, e\right) .
\end{gathered}
$$

The second term can be estimated from above:

$$
\begin{aligned}
& \quad\left|\int_{\Omega} e(t) g(|\xi e(t)+h(t)|) d t\right| \\
& \leq c_{4} \int_{\Omega}|e(t)| d\left(c_{5} \xi|e(t)|\right) d t+c_{6} \chi\left(c_{6} \xi^{-1}, e\right) .
\end{aligned}
$$


The combination of these two estimates together with (19) and the conditions of Theorem 2 imply the estimate $\xi \leq c_{7}$.

Estimate (20) was considered in [3] for various general cases. Here we sketch the proof. Let us split the set $\Omega$ into two parts:

and

$$
\Omega_{1}=\left\{t \in \Omega:|e(t)|>\frac{c_{2}+u_{0}}{\xi}\right\}
$$

$$
\Omega_{2}=\left\{t \in \Omega:|e(t)| \leq \frac{c_{2}+u_{0}}{\xi}\right\}
$$

where the constant $c_{2}$ comes from (17). Since $\operatorname{sign} x(t)=\operatorname{sign} e(t)$ for $t \in \Omega$, we have

$$
\begin{gathered}
\int_{\Omega_{1}} e(t) f(t, x(t)) d t=\int_{\Omega_{1}}|e(t)| f(t, x(t)) \operatorname{sign} e(t) d t \\
\quad=\int_{\Omega_{1}}|e(t)| f(t, x(t)) \operatorname{sign} x(t) d t \\
\geq \int_{\Omega_{1}}|e(t)| \varphi(t,|x(t)|) d t \\
\geq \int_{\Omega_{1}}|e(t)| \varphi\left(t, u_{*}+\xi|e(t)|\right) d t \\
\geq \int_{\Omega}|e(t)| \varphi\left(t, u_{*}+\xi|e(t)|\right) d t-c_{8} \operatorname{mes} \Omega_{2} .
\end{gathered}
$$

Inequality (20) now follows from the estimate mes $\Omega_{2} \leq \chi\left(c_{9} / \xi, e\right)$.

Let us now obtain the estimate (21). Since

by assumption, then

$$
\int_{\Omega} e(t) g(|\xi e(t)|) d t=0
$$

$$
\begin{aligned}
& \left|\int_{\Omega} e(t) g(|\xi e(t)+h(t)|) d t\right| \\
= & \left|\int_{\Omega} e(t) g(|\xi e(t)+h(t)|) d t-\int_{\Omega} e(t) g(|\xi e(t)|) d t\right| \\
\leq & \int_{\Omega}|e(t)| \cdot|g(|\xi e(t)+h(t)|)-g(|\xi e(t)|)| d t \\
& \leq c_{10} \operatorname{mes} \Omega_{2}+c_{11} \int_{\Omega}|e(t)| d\left(c_{12} \xi|e(t)|\right) d t \\
& \leq c_{10} \chi\left(\frac{c_{9}}{\xi}, e\right)+c_{11} \int_{\Omega}|e(t)| d\left(c_{12} \xi|e(t)|\right) d t .
\end{aligned}
$$




\section{Proof of Theorem 1}

Rewrite equation (1) as $x^{\prime \prime}+\left(n^{2}+1\right) x=x+g(|x|)+f(t, x)+b(t)$ and invert the differential operator $\ell$ on the left-hand side with $2 \pi$-periodic boundary conditions, putting $A=\mathcal{L}^{-1}$. The operator $A$ satisfies all of the assumptions of Theorem 2 and is, moreover, self-adjoint rather than just normal. Equation (1) has the equivalent form $\Upsilon x=0$. Any zero $x \in L^{2}$ of the vector field $\Upsilon x$ is obviously twice continuously differentiable. Now if a vector field has non-zero index at infinity, then it has at least one zero. Hence Theorem 1 follows directly from Theorem 2 and under the stated assumptions the index equals \pm 1 .

We need to check that the conditions of Theorem 2 are satisfied under the assumptions of Theorem 1, that is, to check that condition (5) guarantees that both assumptions (13) and (14) for $E_{0}=\{e(t)=A \sin (n t+\theta)\}$ hold and that (6) is valid.

First note that

$$
c_{1} \delta \leq \chi(\delta, e) \leq c_{2} \delta, \quad e \in E_{0}, \quad\|e\|=1, \quad \delta \leq \delta_{0}
$$

so condition (14) can be rewritten as

$$
\int^{\infty} u \varphi(u)=\infty .
$$

By assumption $d(r)=c r^{-\alpha}$, condition (13) has the form of condition (50 which is in fact, more restrictive than (22). The actual calculations here are straightforward, so we shall omit them.

Identity $(6)$ for $e(t)=\sin (n t+\theta)$ follows from the relationships

$$
\begin{aligned}
& \int_{0}^{2 \pi} e(t) g(|e(t)|) d t=\frac{1}{n} \int_{0}^{2 n \pi} \sin \tau g(|\sin \tau|) d \tau \\
= & \int_{0}^{2 \pi} \sin \tau g(|\sin \tau|) d \tau \\
= & \int_{0}^{\pi} \operatorname{sing} \tau g(|\sin \tau|) d \tau+\int_{\pi}^{2 \pi} \sin \tau g(|\sin \tau|) d \tau \\
= & \int_{0}^{\pi} \sin \tau g(|\sin \tau|) d \tau-\int_{0}^{\pi} \sin \tau g(|\sin \tau|) d \tau=0 .
\end{aligned}
$$

\section{Other Applications of Theorem 2}

The first application of Theorem 2 was Theorem 1. In this section, we give two other kinds of examples: solvability results and a theorem on asymptotic bifurcation points.

Consider for some integer $n \neq 0$, the boundary value problem

$$
x^{\prime}+4 n^{2} x=f(t, x)+g(|x|)+b(t), \quad x(0)=x(\pi)=0 .
$$


Theorem A1: Suppose that the functions $f(t, x), g(u)$ and $b(t)$ are bounded and continuous, that one of the one-sided estimates (2) or (3) is valid, that

$$
\int_{0}^{\pi} \sin n t b(t) d t=0
$$

that the function $g(u)$ satisfies Hypothesis $(B)$ for some $\alpha \in(0,1)$, and that $\varphi(u)$ satisfies $(t)$ with this $\alpha$. Then problem (23) has at least one solution.

Note that Theorem A1 is not valid for equation (23) with $(2 n-1)^{2}$ instead of $4 n^{2}$ on the left-hand side.

In the remainder of this section, we consider a special type of ordinary differential equations which arise in control theory:

$$
L\left(\frac{d}{d t}\right) x(t)=M\left(\frac{d}{d t}\right)(f(t, x)+g(|x|)+b(t))
$$

where $L(p)$ and $M(p)$ are real co-prime polynomials with $l=\operatorname{deg} L(p)>m=$ $\operatorname{deg} M(p)$. If $M(p) \equiv 1$, then equation (24) is a usual quasilinear ODE of higher order, but all results below are new even for this case.

Such equations for nonsmooth functions on the right-hand side of (24) can be interpreted in a number of different ways. The usual ones are to consider an equivalent differential equation of first order or an equivalent integral equation (for the periodic problem). Details can be found in almost any text on control theory.

Theorem A2: Suppose that the polynomial $L(p)$ has exactly one pair of roots of the type ni, i.e. + ni and -ni. Under the assumptions of Theorem 1 equation (24) has at least one $2 \pi$-periodic solution.

Now consider equation (24) with a parameter:

$$
L\left(\frac{d}{d t}\right) x(t)=M\left(\frac{d}{d t}\right)(f(t, x ; \lambda)+g(|x| ; \lambda)+b(t ; \lambda))
$$

The following results concerns asymptotic bifurcation points. The general definition given by Mark Krasnosel'skii in the early 1950's can be found in [6]. We reformulate the definition here for the $2 \pi$-periodic problem for equation (25).

The value $\lambda_{0}$ is called the point of nonlinear resonance for equation (25) if for any $\epsilon>0$ there exist a $\lambda$ with $\left\|\lambda-\lambda_{0}\right\| \leq \epsilon$ such that equation (25) for this $\lambda$ has at least one $2 \pi$-periodic solution with amplitude greater than $\epsilon^{-1}$.

Theorem A3: Let $f(t, x ; \lambda) \rightarrow 0$ as $|x| \rightarrow \infty$ for any $\lambda$ sufficiently close to $\lambda_{0}$. Suppose that for $\lambda$ sufficiently close to $\lambda_{0}$, the right-hand side of (25) satisfies all the assumptions of Theorem 1 and that $b_{n}\left(\lambda_{0}\right)=0$ with $b_{n}(\lambda) \neq 0$ for $\lambda \neq \lambda_{0}$ where

$$
b_{n}(\lambda)=\int_{0}^{2 \pi} e^{i n t} b(t ; \lambda) d t .
$$

Then $\lambda_{0}$ is a point of nonlinear resonance for equation (25) and for $\lambda=\lambda_{0}$ there exists at least one $2 \pi$-periodic solution of $(25)$.

We give a brief sketch of the proof here. Suppose (without loss of generality) that the polynomial $L(p)+M(p)$ has no roots of the type $k i$ and consider the equivalent integral equation 


$$
x=A(x+f(t, x ; \lambda)+g(|x| ; \lambda)+b(t ; \lambda)) \equiv A F(t, x ; \lambda) .
$$

Here $A$ is an integral operator and its kernel is generated by the impulse response function of the linear link with the transfer function $W(p)=M(p) /(M(p)+L(P))$. For any integrable function $z(t)$ the image $x(t)=A z(t)$ is the unique $2 \pi$-periodic solution of the linear equation

$$
L\left(\frac{d}{d t}\right) x(t)=M\left(\frac{d}{d t}\right) z(t)
$$

The index at infinity of the vector field $\Psi x=x-A F(t, x ; \lambda)$ for $\lambda=\lambda_{0}$ is equal \pm 1 and the same index for $\lambda \neq \lambda_{0}$ is zero. The last fact (which is non-trivial and follows from $f(t, x ; \lambda) \rightarrow 0$ as $|x| \rightarrow \infty)$ was proved in [4].

The first statement follows directly from the principle of changing index [6], while the second follows in the usual way. For $\lambda=\lambda_{0}$, the index is non-zero, which ensures the existence solutions for values of $\lambda$ sufficiently close to $\lambda_{0}$ in a common ball. The rotation of vector field $\Psi x$ on the boundary of this ball is nonzero, but the index at infinity of this field is zero and hence the other asserted solution exists.

Theorem A3 can be generalized to the case where the polynomial $L(p)$ has $2 n$ $(n>1)$ roots of the type $k i$. Then, instead of $(5)$, it is necessary to suppose the more restrictive condition

$$
\lim _{u \rightarrow \infty} u^{\gamma} \varphi(u)=\infty \text { where } \gamma=\min \left\{\frac{1}{2 n-1}, \alpha\right\} .
$$

More details about the estimate of distribution functions for this case can be found in $[3]$.

\section{References}

[1] Fučik, S., Solvability of Nonlinear Equations and Boundary Value Problems, Society of Czechoslovak Mathematicians and Physicists, Prague 1980.

[2] Krasnosel'skii, A.M., On a method of analysis of resonance problems, Nonlinear Analysis 16:4 (1991), 321-345.

[3] Krasnosel'skii, A.M., Asymptotics of Nonlinearities and Operator Equations, Birkhäuser-Verlag, Basel 1995.

[4] Krasnosel'skii, A.M. and Krasnosel'skii, M.A., Vector fields in a product of spaces and applications to differential equations, Diff. Uravnenija 33:1 (1997), 60-67. (In Russian.)

[5] Krasnosel'skii, A.M. and Mawhin, J., The index at infinity of some twice degenerate compact vector field, Discrete and Contin. Dyn. Sys. 1:2 (1995), 207216.

[6] Krasnosel'skii, M.A. and Zabreiko, P.P., Geometric Methods of Nonlinear Analysis, Springer-Verlag, Heidelberg 1984.

[7] Mawhin, J., Landesman-Lazer's type problems for nonlinear equations, Confer. Semin. Math. Univ. Bari 147 (1977).

[8] Mawhin, J. and Willem, M., Critical Point Theory and Hamiltonian Systems, Springer-Verlag, Heidelberg 1989. 


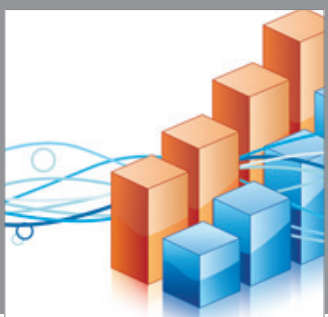

Advances in

Operations Research

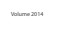

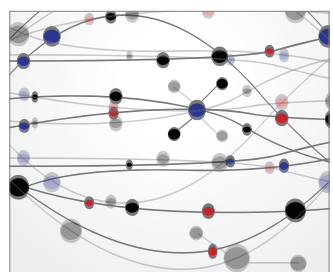

\section{The Scientific} World Journal
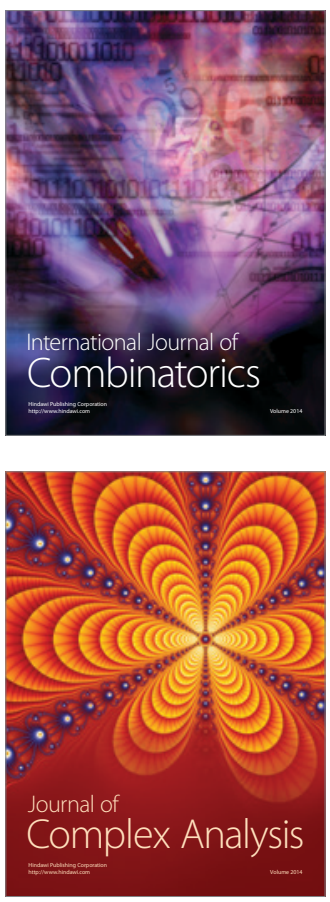

International Journal of

Mathematics and

Mathematical

Sciences
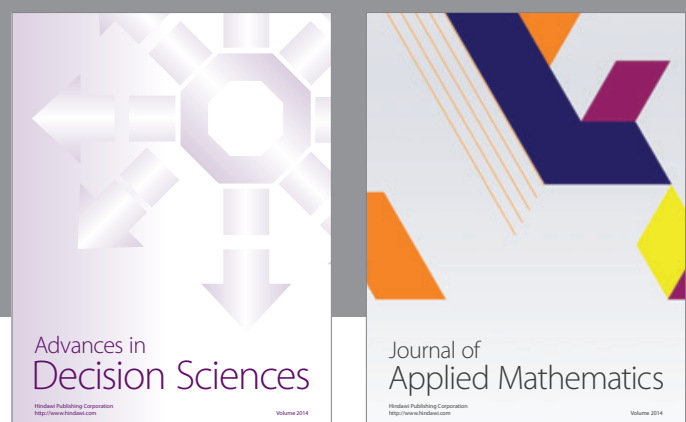

Journal of

Applied Mathematics
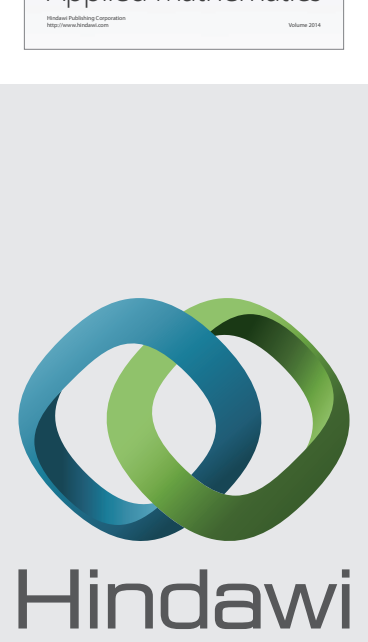

Submit your manuscripts at http://www.hindawi.com
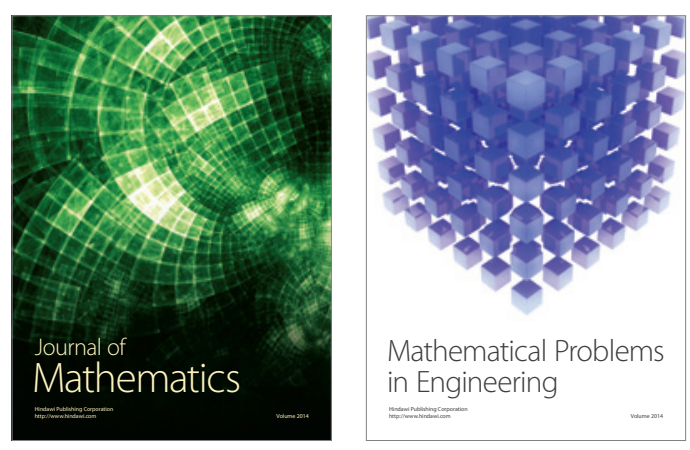

Mathematical Problems in Engineering
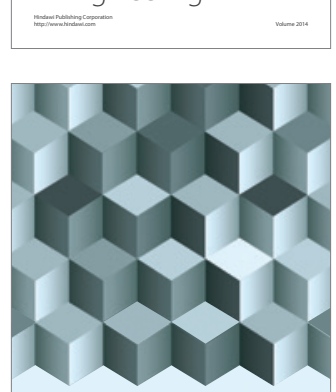

Journal of

Function Spaces
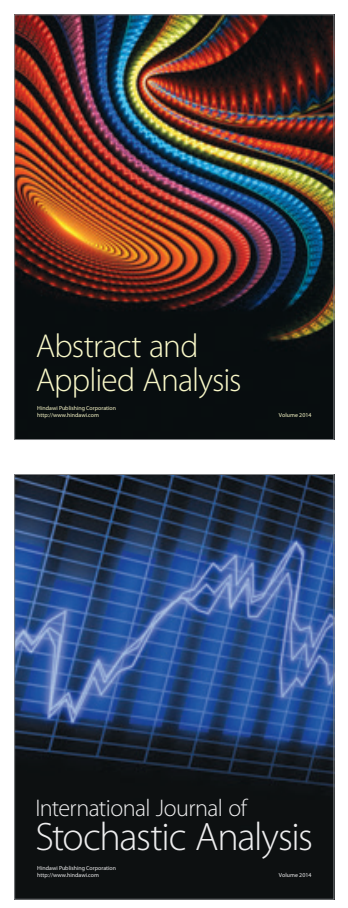

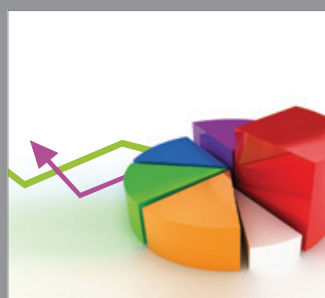

ournal of

Probability and Statistics

Promensencen
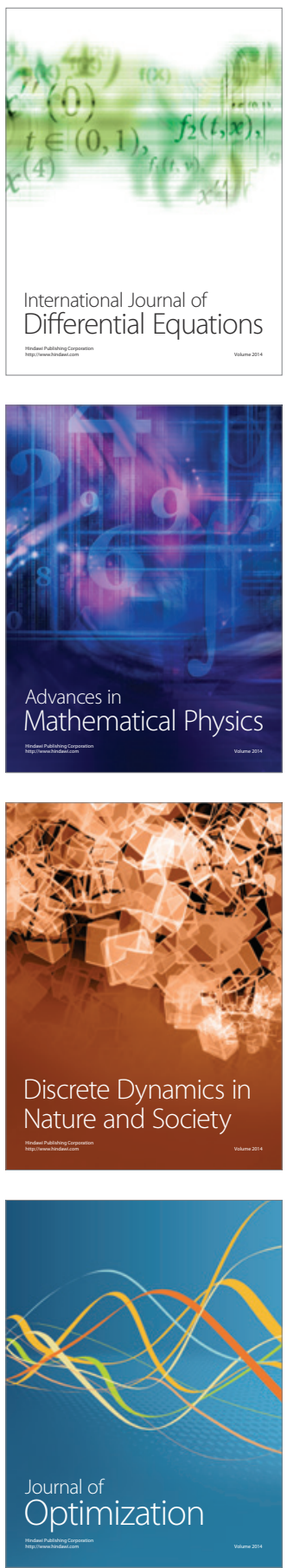